\title{
Morel-Lavallee Lesion of the Lower Back Mimicking an Abscess: A Case Report
}

\author{
Sandra de Montbrun ${ }^{*}$, Korosh Khalili, Steven MacLellan, Alexandra Easson \\ Mount Sinai Hospital, University of Toronto, Toronto, Canada \\ Email: *sandra.demontbrun@utoronto.ca
}

Received December 2, 2011; revised January 31, 2012; accepted February 20, 2012

\begin{abstract}
A closed degloving injury, or Morel-Lavallee lesion, is the result of a severe, traumatic, shearing injury, causing separation of the skin and subcutaneous tissue from the underlying deep fascia. Though well described in the orthopedic trauma literature, this lesion is not well recognized by the general surgeon in the poly-trauma setting. We present a case of a 41 year old man who was referred to the general surgery service for a "back abscess". Upon patient interview, history of a recent motor vehicle collision (MVC) was obtained, including a pelvic fracture. Imaging demonstrated a large Morel-Lavellee lesion extending from the pelvis into the lower back. Knowledge of this entity is crucial to avoid unnecessary procedures in the management of these patients.
\end{abstract}

Keywords: Closed Degloving Injury; Morel-Lavallee Lesion; Trauma; Case Report

\section{Introduction}

Closed degloving injury was first described in the mid19th century by Morel-Lavallee [1] and his name is the eponym attached to this clinical entity. A Morel-Lavallee lesion is the result of a violent, traumatic tangential force that causes separation of the skin and subcutaneous tissue from the underlying deep fascia [2] and is often the result of a high energy, blunt pelvic or acetabular trauma. While well described in the orthopaedic trauma literature, this entity is less known to the general surgeon despite its ability to mimic a general surgical problem. Thus it is important for the general surgeon to be aware of the Morel-Lavallee lesion and its clinical significance. In this report we present the case of a patient referred to the general surgery service with the incorrect diagnosis of a back abscess.

\section{Case Presentation}

A 41 year old man presented to the emergency department (ER) of a downtown teaching hospital with sacral swelling that had significantly increased in size over the past week. For the past 5 days he had increasing pain over the area, as well as fever and chills. On physical exam he was afebrile, and his vital signs were stable. A large fluctuant swelling spanned the entire lower back and was painful, warm, erythematous and blanched with pressure. Blood work was normal; white blood cells

${ }^{*}$ Corresponding author.
(WBC) were mildly elevated (10.29). An ultrasound showed an extensive subcutaneous complex collection. General surgery was consulted for drainage of a back abscess (Figure 1(a)).

On further interview, it appeared that 3 months prior he was in a serious MVC which resulted in admission to another major trauma center. His injuries included a fractured rib, pelvis and clavicle as well as a brain injury. None of his injuries required surgical management and he remained hospitalized for one month, mainly to recover from his brain injury. He had no other significant past medical or surgical history.

Given this history, a CT scan was performed which demonstrated a large fluid collection $(22 \times 4.5 \times 16.5 \mathrm{~cm})$ superficial to the gluteus maximus on the right extending across the midline to the left side (with some extension of the collection into the gluteus muscle caudally) (Figures 1(b)-(c)). A radiological diagnosis of a MorelLavallee lesion was made.

The patient was admitted to the general surgical service. A percutaneous drain (12 French catheter) placed by interventional radiology immediately drained 650 сс of serous fluid. Over the following 2 days the drain put out 700 cc of serous fluid. Cultures were negative. The patient was sent home 2 days later with his drain in place.

The patient was seen in follow-up 1 month later with approximately $100 \mathrm{cc} /$ day output. A sinogram demonstrated a residual cavity, so the drain was left in. The drainage stopped several days later and the drain was 


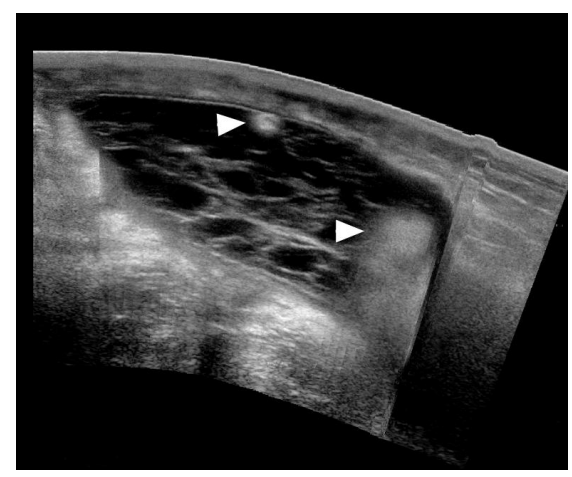

(a)

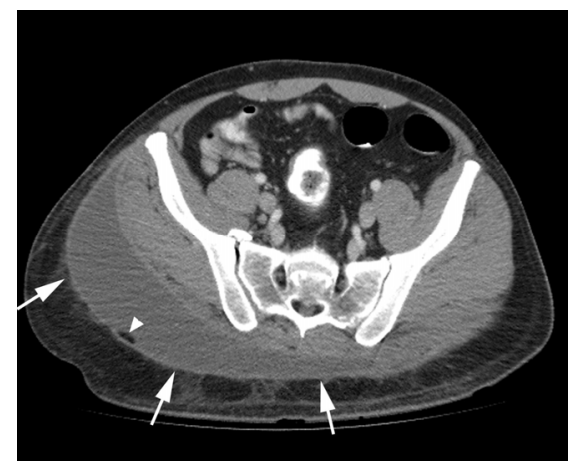

(b)

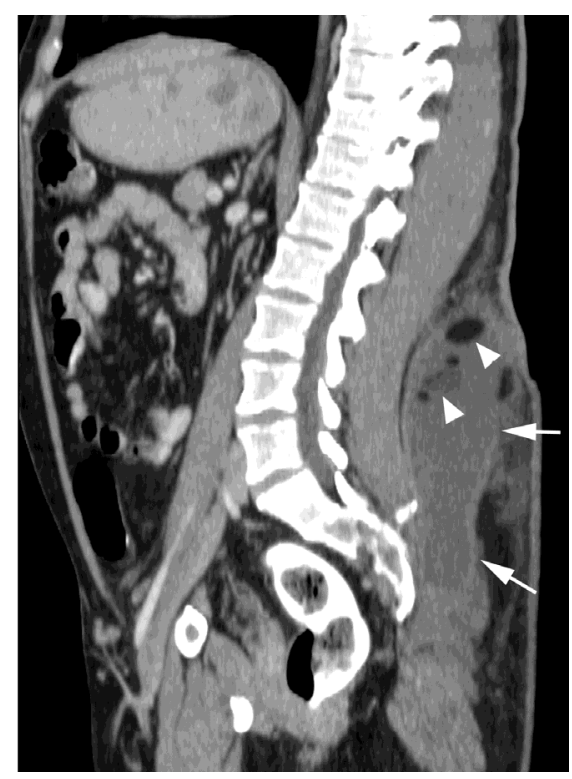

(c)

Figure 1. (a) Transverse ultrasound demonstrating cystic collection with multiple internal septations and echogenic foci (arrowheads) representing internal fatty debris; (b) Axial CT of the capsulated Morel-Lavallee lesion (arrows) located between the subcutaneous fat and investing fascia of gluteal and lumbar muscles. Note internal fatty debris (arrowheads), typical of this lesion; (c) Sagittal reformatted CT demonstrating the cranio-caudal extent of the lesion (arrows) and internal fatty debris (arrowheads). Caudally the lesion extended into the muscular fibers of the gluteal muscles. removed. After 2 years, the patient has had no further problems and no recurrence of his Morel-Lavallee lesion.

\section{Discussion}

\subsection{Pathophysiology}

The Morel-Lavallee lesion is a traumatic closed internal degloving injury which was initially described in 1863 by Morel-Lavallee [1]. In 1993, the term "Morel-Lavallee” lesion was used in a classification system for acetabular fractures to specifically refer to a degloving injury over the area of the greater trochanter. Now, the eponym has been used to describe closed degloving injuries in other anatomical locations. It most commonly occurs over the greater trochanter, but can occur anywhere over the trunk, buttock or thigh [3]. It is most frequently associated with high energy pelvic and acetabular trauma [4]. The traumatic event leads to avulsion of the fat and subcutaneous tissue from the underlying deep fascia resulting in an avascular cavity. This cavity then fills with hematoma and liquefied fat, creating the lesion [5]. The vascular supply to the overlying skin becomes compromised from the trauma since the only remaining blood supply to the skin is the subdermal plexus [6]. This makes the skin susceptible to ischemia and necrosis. The major concern with these injuries is the associated high incidence of deep bone and soft tissue infection which can lead to sepsis and serious disability [2,7].

\subsection{Diagnosis}

The diagnosis is based on physical examination and an associated history of trauma. A Morel-Lavallee lesion can present anywhere from days to months after the initial injury and may or may not be associated with an underlying pelvic or acetabular fracture. The hallmark physical finding is the presence of a soft fluctuant area, [3] as illustrated in our case. The overlying skin may demonstrate signs of injury, loss of cutaneous sensation and hypermobility.

\subsection{Imaging}

Ultrasound, MRI and CT have been used to diagnose Morel-Lavallee lesions. On all three, the lesions are fusiform or ovoid, located between the hypodermis and the deeper fascial planes. The appearance of internal contents of the lesion and the formation of a capsule depend on its age. Lesions under 6 months will be complex cysts filled with debris and blood products. Older lesions become clear cystic spaces, with a discernable capsule [8]. Ultrasound can locate and determine the extent of the lesion. The lesion appears as a complex cystic mass with internal septations early on and evolves into an anechoic cyst compatible with liquefied hematoma [8]. MRI can 
characterize the internal contents as blood products or seroma, helping differentiate this lesion from a soft tissue mass [9]. Three-dimensional CT reconstructions are now routinely being used by some authors to screen for Morel-Lavallee lesions in patients sustaining traumatic pelvic and acetabular fractures [4]. The demonstration of fatty debris representing fat necrosis, as seen in our patient (Figure 1), is a typical finding on all three modalities.

\subsection{Treatment}

Several different treatment modalities have been described in the literature for closed internal degloving injuries. In the acute traumatic setting Hak et al. have suggested thorough surgical debridement in order to prevent infection [3]. Other methods that have been described include aspiration with or without pressure therapy, injection of a sclerosing agent and prolonged closed surgical drainage [10]. Our case demonstrates successful treatment with a minimally invasive percutaneous approach.

\section{Conclusion}

Closed degloving injuries are an uncommon clinical entity, [3] and more familiar to the orthopaedic surgeon than the general surgeon. A Morel-Lavallee lesion may easily be referred to the general surgery service as a soft tissue mass or soft tissue infection as it may occur long after the other trauma effects have healed. The current case of a Morel-Lavallee lesion was referred to the general surgery service with the presumptive diagnosis of a back abscess. Once the history of trauma was elicited and radiological nuances were identified, the diagnosis of a Morel-Lavallee lesion was made and the patient was able to undergo successful percutaneous drainage. It is critical for the general surgeon to be aware of this clinical entity so that it is treated appropriately, realizing its clinical significance.

\section{Author Declaration}

We the undersigned declare that this manuscript is original, has not been published before and is not currently being considered for publication elsewhere.

We confirm that there are no known conflicts of interest associated with this publication and there has been no significant financial support for this work that could have influenced its outcome.

We confirm that the manuscript has been read and approved by all named authors.

We confirm that any aspect of the work covered in this manuscript that has involved either experimental animals or human patients has been conducted with the ethical approval of all relevant bodies and that such approvals are acknowledged within the manuscript.

We understand that the Corresponding Author is the sole contact for the Editorial process responsible for communicating with the other authors about progress, submissions of revisions and final approval of proofs.

\section{REFERENCES}

[1] Morel-Lavallee, "Decollements Traumatique de la Peau et des Couches Sous-Jacented," Archives of General Medicine, Vol. 1, 1863, pp. 20-38.

[2] T. J. Phillips, B. Jeffcote and D. Collopy, "Bilateral Morel-Lavallée Lesions after Complex Pelvic Trauma: A Case Report," The Journal of Trauma, Vol. 65, No. 3, 2008, pp. 708-711. doi:10.1097/TA.0b013e31815eb74b

[3] D. J. Hak, S. A. Olson and J. M. Matta, "Diagnosis and Management of Closed Internal Degloving Injuries Associated with Pelvic and Acetabular Fractures: The MorelLavallée Lesion,” The Journal of Trauma, Vol. 42, No. 6, 1997, pp. 1046-1051. doi:10.1097/00005373-199706000-00010

[4] R. N. Reddix, E. Carroll and L. X. Webb, "Early Diagnosis of a Morel-Lavallee Lesion Using Three-Dimensional Computed Tomography Reconstructions: A Case Report," The Journal of Trauma, Vol. 67, 2009, pp. E57E59. doi:10.1097/TA.0b013e31814b2d20

[5] M. Tile, D. Helfet and J. Kellam, "Fractures of the Pelvis and Acetabulum,” 3rd Edition, Lippincott, Williams \& Wilkins, Philadelphia, 2003.

[6] S. Tseng and P. Tornetta III, "Percutaneous Management of Morel-Lavallee Lesions," The Journal of Bone and Joint Surgery. American Volume, Vol. 88, No. 1, 2006, pp. 92-96. doi:10.2106/JBJS.E.00021

[7] H. L. Lin, W. C. Lee, L. C. Kuo and C. W. Chen, "Closed Internal Degloving Injury with Conservative Treatment," The American Journal of Emergency Medicine, Vol. 26, 2008, pp. 254.e5-6.

[8] C. Neal, J. A. Jacobson, C. Brandon, M. Kalume-Brigido, Y. Morag and G. Girish, "Sonography of Morel-Lavallee Lesions," Journal of Ultrasound in Medicine, Vol. 27, No. 7, 2008, pp. 1077-1081.

[9] J. M. Mellado, L. Pérez del Palomar, L. Díaz, A. Ramos and A. Saurí, "Long-Standing Morel-Lavallée Lesions of the Trochanteric Region and Proximal Thigh: MRI Features in Five Patients," American Journal of Roentgenology, Vol. 182, No. 5, 2004, pp. 1289-1294.

[10] S. A. Kottmeier, S. C. Wilson, C. T. Born, G. A. Hanks, W. M. Iannacone and W. G. De Long, "Surgical Management of Soft Tissue Lesions Associated with Pelvic Ring Injury," Clinical Orthopaedics and Related Research, Vol. 329, 1996, pp. 46-53. doi:10.1097/00003086-199608000-00007 